\title{
Global Calibration Method of a Camera Using the Constraint of Line Features and 3D World Points
}

\author{
Guan $\mathrm{Xu}^{1}$, Xinyuan Zhang ${ }^{1}$, Xiaotao $\mathrm{Li}^{2}$, Jian $\mathrm{Su}^{1}$, and Zhaobing Hao ${ }^{1}$ \\ ${ }^{1}$ Department of Vehicle Application Engineering, Traffic and Transportation College, Nanling Campus, Jilin University, \\ Renmin Street 5988\#, 130025, Changchun, Jilin, P. R. China, xuguan@jlu.edu.cn \\ ${ }^{2}$ Mechanical Science and Engineering College, Nanling Campus, Jilin University, Renmin Str. 5988\#, 130025, Changchun, \\ Jilin, P. R. China, lixiaotao@jlu.edu.cn
}

\begin{abstract}
We present a reliable calibration method using the constraint of 2D projective lines and 3D world points to elaborate the accuracy of the camera calibration. Based on the relationship between the 3D points and the projective plane, the constraint equations of the transformation matrix are generated from the 3D points and 2D projective lines. The transformation matrix is solved by the singular value decomposition. The proposed method is compared with the point-based calibration to verify the measurement validity. The mean values of the root-mean-square errors using the proposed method are $7.69 \times 10^{-4}, 6.98 \times 10^{-4}, 2.29 \times 10^{-4}$, and $1.09 \times 10^{-3}$ while the ones of the original method are $8.10 \times 10^{-4}, 1.29 \times 10^{-2}, 2.58 \times 10^{-2}$, and $8.12 \times 10^{-3}$. Moreover, the average logarithmic errors of the calibration method are evaluated and compared with the former method in different Gaussian noises and projective lines. The variances of the average errors using the proposed method are $1.70 \times 10^{-5}, 1.39 \times 10^{-4}, 1.13 \times 10^{-4}$, and $4.06 \times 10^{-4}$, which indicates the stability and accuracy of the method.
\end{abstract}

Keywords: Calibration method, projective lines, measurement validity, Gaussian noises.

\section{INTRODUCTION}

Camera calibration is an important source of the correspondence information between $3 \mathrm{D}$ space and 2D image [1]-[3]. The task of a camera calibration method is to provide the geometric transformation from the $2 \mathrm{D}$ points in an image to the 3D coordinates in the space [4], [5]. Therefore, the camera calibration is the foundation for the optical test and measurement. It has extensive applications such as object identification, image measurement, robot navigation, vehicle profile reconstruction [6]-[10], etc.

Traditional camera calibration method establishes the constraint relationship between the standard object and the image to calculate the parameters of a camera [11], [12]. Later in Zhang's work, feature points for the calibration are generated from the photos of the calibration object. The calibration object is a $1 \mathrm{D}$ bar that rotates around a fixed point [13]. The shortcoming of the $1 \mathrm{D}$ calibration is that the 1D object should be exactly restrained to perform the controlled special motions, such as planar motions or rotations around a fixed point [14]. The 2D plane-based calibration requires a planar pattern shown at a few different orientations. The constraint equations are built by the properties of the unit orthogonal matrix to solve the camera parameters [15]. It is flexible and easy to implement in the laboratory, however, the calibration precision is limited by the measurement distance and the number of captured images. Although both existing $1 \mathrm{D}$ and 2D calibration methods are easier to apply than 3D methods, for the reconstruction applications the methods require the appropriate measurement distance to achieve the higher precision [16], [17]. A calibration method is built by the geometrical model between the 3D coordinates of the laser stripe on the target and the 2D coordinates in the image [18]. Cui proposed a precise calibration method for a binocular vision system that is devoted to minimizing the distance error between the reconstructed point through optimal triangulation and the ground truth in a 3D measurement coordinate system [19]. The drawback of the above point-based methods is the accuracy of the calibration method declines when the locations of measured feature points are affected by noises.

It is well-known that in practice it is difficult to accurately and reliably extract all the features in all images considering the noises, image blur, and different illumination [20]. Large noises, outliers, missing features, and mismatches lead to the inaccurate calibration result [21]. Therefore, it is important to provide a more stable and precise camera calibration method. We raise the global calibration method of a camera using the constraint of 2D projective lines and 3D world points. The Hough transform is adopted to extract the 2D projective lines in the image, which provides a high robustness even for large occlusions or bad lighting conditions [22]. The camera calibration is implemented by the relationship of the known $3 \mathrm{D}$ points in the real world 
and the precise lines' coordinates in the image. In most applied measurements, straight line is the attractive object because it is little affected by noises. Compared with traditional calibration methods with feature points, the line-based camera calibration method has better noise-immunity and higher accuracy. It provides a stable solution of the camera calibration problem in a convenient and accurate way. It is also possible to reduce the effect of image noises on the accuracy of the inferred camera parameters.

A reliable calibration method is explored in this paper, which elaborates the accuracy of the calibration. A projective line in the image is determined by the corresponding 3D points and the projective plane. According to the geometrical relationship between $3 \mathrm{D}$ points and $2 \mathrm{D}$ projective lines, a group of $2 \mathrm{D}$ projective lines is typically extracted to obtain the constraints of the transformation matrix $\mathbf{P}$ of the camera. Meanwhile, the proposed method is compared with the original method of $3 \mathrm{D}$ points and $2 \mathrm{D}$ projective points to verify the measurement validity and the noise immunity.

\section{LINE-BASED CALIBRATION METHOD}

In the camera model in Fig.1., the world coordinate system $O-X Y Z$ is attached to the $3 \mathrm{D}$ calibration board. $o-x y$ is the image coordinate system. $o-u v w$ is the camera coordinate system. One 3D line $\mathbf{L}_{i}$ and two 3D points $\mathbf{X}_{i}^{(j)} \quad(j=1,2)$ on the $3 \mathrm{D}$ line $\mathbf{L}_{i}$ in the world coordinate system $O-X Y Z$ are projected to the $2 \mathrm{D}$ image plane $o-x y$. The generated geometrical elements are the 2D line $\mathbf{l}_{i}$ and two 2D points $\mathbf{x}_{i}^{(j)}$, respectively. We choose two 3D points on each line because more than two points depend on the two 3D points, which means the points can be expressed by the former two $3 \mathrm{D}$ points. The relationship between a 3D point $\mathbf{X}_{i}^{(j)}$ and a 2D point $\mathbf{x}_{i}^{(j)}$ from the pinhole camera model is [23], [24]

$$
\mathbf{P X}_{i}^{(j)}=\mathbf{X}_{i}^{(j)}
$$

where $\mathbf{P}=\left[p_{\mathrm{m}, \mathrm{n}}\right]_{3 \times 4}$ is the projective matrix of the camera, $\mathbf{X}_{i}^{(j)}=\left[X_{i}^{(j)}, Y_{i}^{(j)}, Z_{i}^{(j)}, 1\right]^{\mathrm{T}}$ is a $4 \times 1$ homogeneous vector of the world coordinate of the $3 \mathrm{D}$ point, and $\mathbf{x}_{i}^{(j)}=\left[x_{i}^{(j)}, y_{i}^{(j)}, 1\right]^{\mathrm{T}}$ is a $3 \times 1$ homogeneous vector of the image coordinate of the $3 \mathrm{D}$ point.

According to the general equation of a 2D line, a 2D point $\mathbf{x}_{i}^{(j)}$ on a $2 \mathrm{D}$ line $\mathbf{l}_{i}$ satisfies

$$
\left(\mathbf{x}_{i}^{(j)}\right)^{\mathrm{T}} \mathbf{l}_{i}=0
$$

where $\mathbf{l}_{i}^{(j)}=\left[l_{i}^{(1)}, l_{i}^{(2)}, 1\right]^{\mathrm{T}}$ is a $3 \times 1$ homogeneous vector composed by the equation coefficients of the general equation of a 2D line. As the equation coefficients determine the position and direction of a $2 \mathrm{D}$ line, the vector $\mathbf{l}_{i}$ stands for a 2D line.

Substituting (1) in (2), then

$$
\left(\mathbf{X}_{i}^{(j)}\right)^{\mathrm{T}} \mathbf{P}^{\mathrm{T}} \mathbf{l}_{i}=0
$$

According to the general equation of a plane, (3) represents a 3D point $\mathbf{X}_{i}^{(j)}$ that is located on a 3D plane $\boldsymbol{\pi}_{i}$. Moreover, the projective plane $\boldsymbol{\pi}_{i}$ is $\mathbf{P}^{\mathrm{T}} \mathbf{l}_{i}$, which is determined by the optical center $o$ of the camera and the 2D projective line $\mathbf{l}_{i}$.

For a line and two points on it, $j=1,2$, (3) can alternatively be written as

$$
\mathbf{A}_{i} \mathbf{p}=\mathbf{0}_{2 \times 1}
$$

where

$$
\begin{aligned}
& \mathbf{A}_{i}=\left[\begin{array}{llllllllllll}
l_{i}^{(1)} X_{i}^{(1)} & l_{i}^{(1)} Y_{i}^{(1)} & l_{i}^{(1)} Z_{i}^{(1)} & l_{i}^{(1)} & l_{i}^{(2)} X_{i}^{(1)} & l_{i}^{(2)} Y_{i}^{(1)} & l_{i}^{(2)} Z_{i}^{(1)} & l_{i}^{(2)} & X_{i}^{(1)} & Y_{i}^{(1)} & Z_{i}^{(1)} & 1 \\
l_{i}^{(1)} X_{i}^{(2)} & l_{i}^{(1)} Y_{i}^{(2)} & l_{i}^{(1)} Z_{i}^{(2)} & l_{i}^{(1)} & l_{i}^{(2)} X_{i}^{(2)} & l_{i}^{(2)} Y_{i}^{(2)} & l_{i}^{(2)} Z_{i}^{(2)} & l_{i}^{(2)} & X_{i}^{(2)} & Y_{i}^{(2)} & Z_{i}^{(2)} & 1
\end{array}\right] \\
& , \mathbf{p}=\left[\begin{array}{lllllllll}
p_{11} & p_{12} & p_{13} & p_{14} & p_{21} & p_{22} & p_{23} & p_{24} & p_{31} \\
p_{32} & p_{33} & p_{34}
\end{array}\right]^{\mathrm{T}} .
\end{aligned}
$$

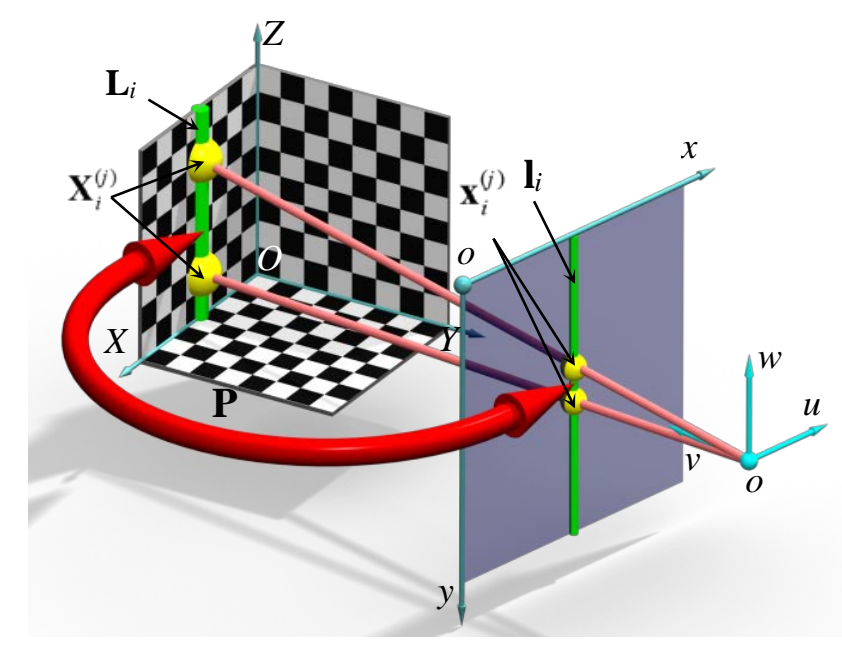

Fig.1. The calibration method adopts 2D projective lines $\mathbf{l}_{i}$ and 3D points $\mathbf{X}_{i}^{(j)}$.

$n$ lines and $2 n$ points on them are typically extracted to obtain the transformation matrix $\mathbf{P}$. It means $i=1,2, \ldots, n$. Linear equations can be obtained from the stacking of (4) [25]

$$
\mathcal{A} \mathbf{p}=\mathbf{0}_{2 n \times 1}
$$

where $\mathcal{A}=\left[\mathrm{A}_{1}^{\mathrm{T}}, \mathbf{A}_{2}^{\mathrm{T}}, \ldots, \mathbf{A}_{n}^{\mathrm{T}}\right]^{\mathrm{T}}, \mathcal{A}$ is derived from the vectors of $2 \mathrm{D}$ lines $\mathbf{l}_{i}$ and the world coordinates of the 3D points $\mathbf{X}_{i}^{(j)}$.

The singular value decomposition of the matrix $\mathcal{A}$ is expressed by [26]

$$
\mathcal{A}=\mathbf{S} \Lambda \mathbf{D}^{\mathrm{T}}
$$

where $\mathbf{S}$ and $\mathbf{D}$ are orthogonal matrices, and $\boldsymbol{\Lambda}$ is a diagonal matrix with the singular values.

From the orthogonal matrix $\mathbf{D}$, we have [26] 


$$
\mathbf{p}=\mathbf{d}^{*}
$$

where $\mathbf{d}^{*}$ is the column vector in the orthogonal matrix $\mathbf{D}$ related to the smallest singular value in $\boldsymbol{\Lambda}$. The block diagram of the calibration method is shown in Fig.2.

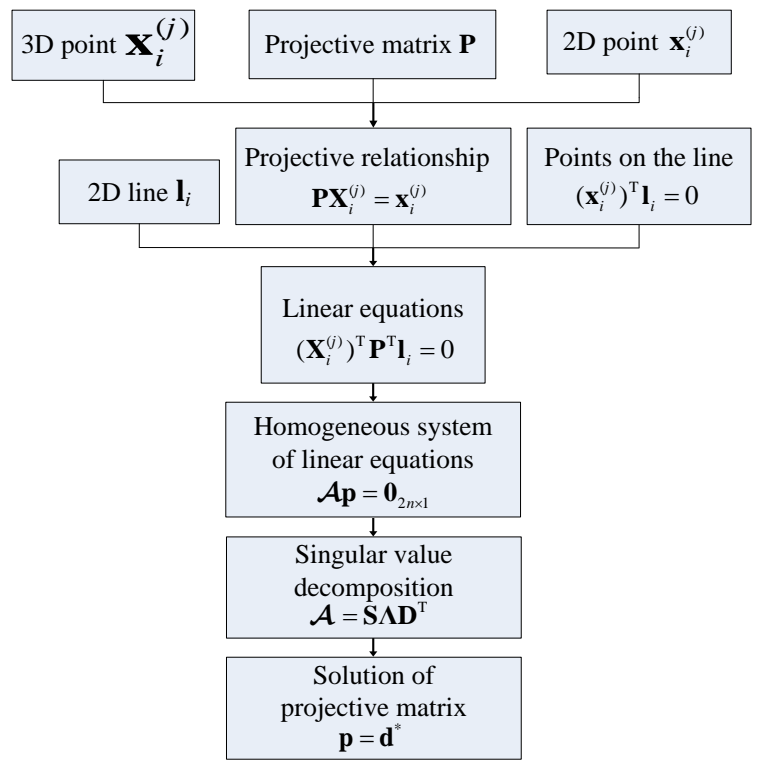

Fig.2. The block diagram of the calibration method using lines and points.

\section{EXPERIMENTS AND DISCUSSIONS}

According to the analysis above, the transformation matrix $\mathbf{P}$ can be generated from $2 n 3 \mathrm{D}$ points $\mathbf{X}_{i}^{(j)}$ and $n 2 \mathrm{D}$ projective lines $\mathbf{I}_{i}$. The original images of a 3D calibration board are shown in Fig.3. The dimension of the 3D calibration board is $500 \mathrm{~mm} \times 500 \mathrm{~mm} \times 500 \mathrm{~mm}$. $8 \times 8$ squares with the dimension of $60 \mathrm{~mm} \times 60 \mathrm{~mm}$ cover the three planes of the calibration board, respectively.
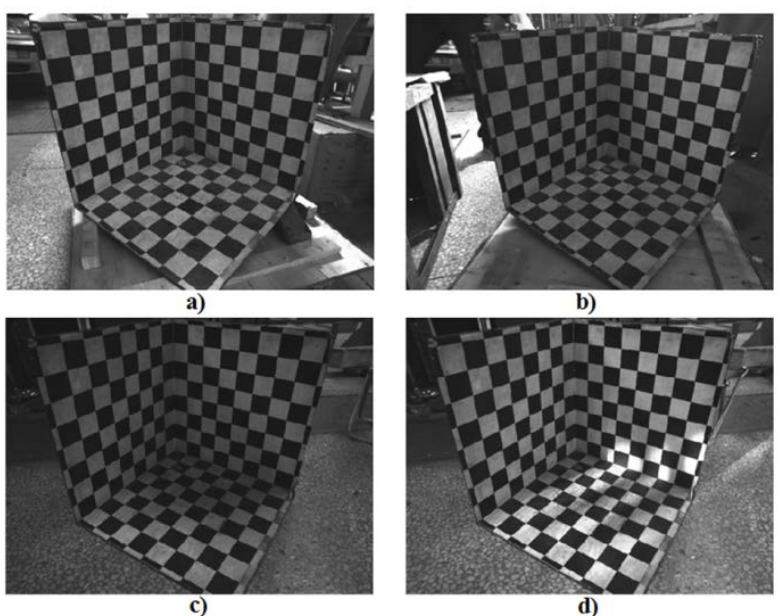

Fig.3. a), b), c), d) are four original images of the calibration board. The views of the camera and the illuminating conditions are different in the four images.
The Hough transform is adopted to extract the precise coordinates of the 2D projective lines [27]. The method aims to find lines within a parameter space of lines by a voting procedure. From the voting procedure, parameter candidates are obtained as local maximal value that is constructed by the Hough transform. The extraction results of the lines on the calibration board are illustrated in Fig.4.a) - Fig.4.d). It is obvious that the lines are recognized by the Hough transform exactly.
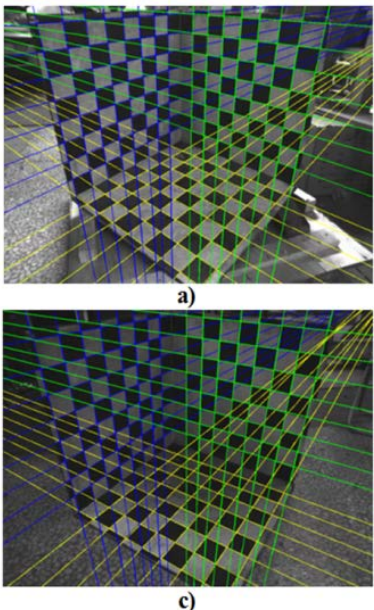

c)
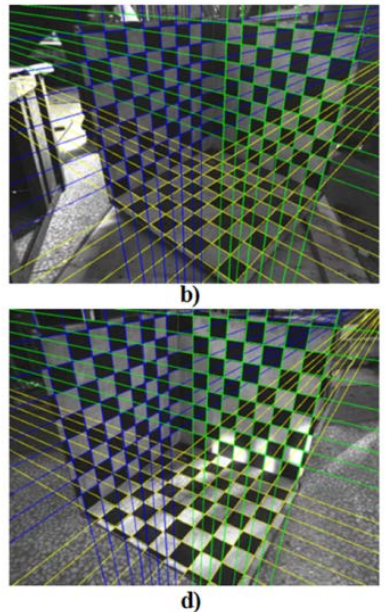

d)
Fig.4. Line recognition results on the three planes of the calibration board. a) the recognized lines of Fig.3.a), b) the recognized lines of Fig.3.b), c) the recognized lines of Fig.3.c), d) the recognized lines of Fig.3.d).

The proposed method is compared with the original method of 3D points and 2D projective points to verify the measurement validity and noise immunity. First, the above transformation matrix $\mathbf{P}_{1}$ of the proposed method is experimentally obtained from (7). The transformation matrix of the original method is denoted by $\mathbf{P}_{\mathrm{p}}$. Then the 3D point $\mathbf{X}_{k}$ is reprojected to the 2D image by [28]

$$
\begin{aligned}
& \mathbf{X}_{\mathrm{lk}}=\mathbf{P}_{1} \mathbf{X}_{k} \\
& \mathbf{X}_{\mathrm{p} k}=\mathbf{P}_{\mathrm{p}} \mathbf{X}_{k}
\end{aligned}
$$

where $\mathbf{x}_{l k}, \mathbf{x}_{\mathrm{p} k}$ are the projective coordinates of the 3D point $\mathbf{X}_{k}$ by the line-based method and point-based method, respectively.

The projective lines coordinates $\mathbf{l}_{\mathbf{l}}, \mathbf{l}_{\mathrm{p} i}$ are generated from the projective coordinates $\mathbf{x}_{k}, \mathbf{x}_{\mathrm{pk}}$ and the least square method. We define the errors of the two methods by [28]

$$
\begin{gathered}
\Delta \mathbf{l}_{\mathrm{li}}=\left\|\mathbf{l}_{\mathrm{li}}-\mathbf{l}_{i}\right\| \\
\Delta \mathbf{l}_{\mathrm{p} i}=\left\|\mathbf{l}_{\mathrm{p} i}-\mathbf{l}_{i}\right\|
\end{gathered}
$$


We carry out experiments on the $512 \times 384$ images to compare the calibration precision of the point-based calibration and the line-based calibration. The accuracy in the calibration is evaluated by the difference between the $2 \mathrm{D}$ projective lines $\mathbf{l}_{l i}, \mathbf{l}_{\mathrm{p} i}$ and the $2 \mathrm{D}$ lines $\mathbf{l}_{i}$ extracted by the Hough transform. 48 lines are adopted to the calibration. The projective lines $\mathbf{l}_{l i}, \mathbf{l}_{\mathrm{p} i}$ are obtained by transformation matrices $\mathbf{P}_{\mathrm{p}}$ and $\mathbf{P}_{\mathrm{l}}$. The experimental errors using the methods are shown in Fig.5.
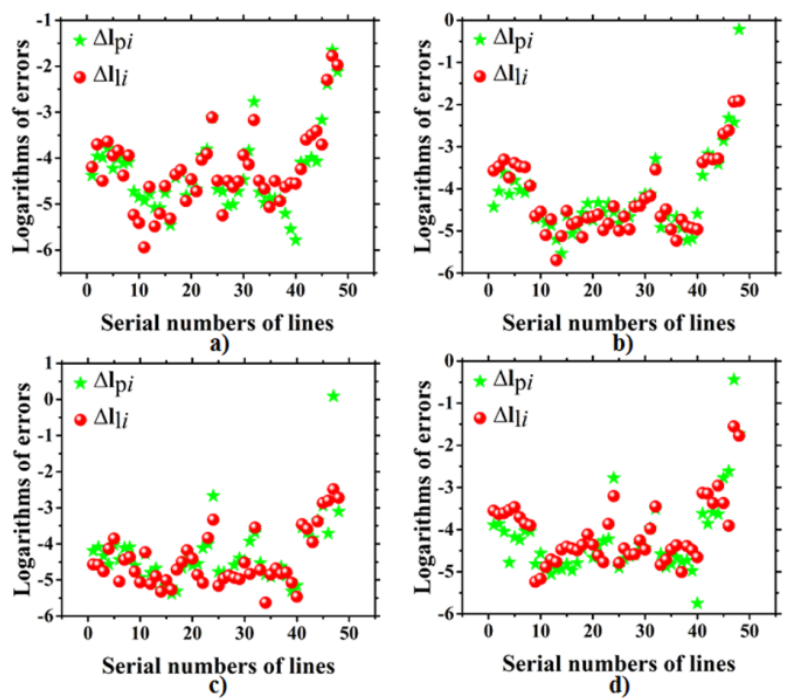

Fig.5. The root-mean-square errors of the projective lines adopting the point-based calibration method and the line-based calibration method. a) the errors of Fig.3.a), b) the errors of Fig.3.b), c) the errors of Fig.3c), d) the errors of Fig.3.d).

Table 1. Results of mean values of root-mean-square errors.

\begin{tabular}{|c|c|c|}
\hline \multirow{2}{*}{ Image } & \multicolumn{2}{|c|}{ Mean values of root-mean-square errors } \\
\cline { 2 - 3 } & Point-based method & Line-based method \\
\hline $\mathrm{a}$ & $8.10 \times 10^{-4}$ & $7.69 \times 10^{-4}$ \\
\hline $\mathrm{b}$ & $1.29 \times 10^{-2}$ & $6.98 \times 10^{-4}$ \\
\hline $\mathrm{c}$ & $2.58 \times 10^{-2}$ & $2.29 \times 10^{-4}$ \\
\hline $\mathrm{d}$ & $8.12 \times 10^{-3}$ & $1.09 \times 10^{-3}$ \\
\hline
\end{tabular}

Table 2. Results of averages of measurement errors.

\begin{tabular}{|c|c|c|c|}
\hline & \multirow[b]{2}{*}{ Image } & \multicolumn{2}{|c|}{ Averages of measurement errors } \\
\hline & & $\begin{array}{l}\text { Point-based } \\
\text { method }\end{array}$ & $\begin{array}{l}\text { Line-based } \\
\text { method }\end{array}$ \\
\hline \multirow{2}{*}{$\mathrm{a}$} & $x$ direction & $2.05 \times 10^{-4}$ & $1.86 \times 10^{-4}$ \\
\hline & $y$ direction & $1.10 \times 10^{-3}$ & $1.05 \times 10^{-3}$ \\
\hline \multirow{2}{*}{ b } & $x$ direction & $1.63 \times 10^{-3}$ & $2.04 \times 10^{-4}$ \\
\hline & $y$ direction & $1.81 \times 10^{-2}$ & $9.48 \times 10^{-4}$ \\
\hline \multirow{2}{*}{ C } & $x$ direction & $5.21 \times 10^{-3}$ & $8.75 \times 10^{-5}$ \\
\hline & $y$ direction & $3.60 \times 10^{-2}$ & $2.99 \times 10^{-4}$ \\
\hline \multirow{2}{*}{ d } & $x$ direction & $1.64 \times 10^{-3}$ & $2.54 \times 10^{-4}$ \\
\hline & $y$ direction & $1.13 \times 10^{-2}$ & $1.49 \times 10^{-3}$ \\
\hline
\end{tabular}

In order to show the experimental results more clearly, we analyze the logarithms of the root-mean-square errors in the $x, y$ directions for different serial numbers of the projective lines. We obtain 48 groups of errors with the two methods for each position of the camera, respectively. The results are compared in Table 1 . and Table 2.

The mean values of root-mean-square errors using point-based method in Fig.5.a) - Fig.5.d) are $8.10 \times 10^{-4}$, $1.29 \times 10^{-2}, 2.58 \times 10^{-2}$, and $8.12 \times 10^{-3}$, respectively. While the mean values using the proposed method are $7.69 \times 10^{-4}$, $6.98 \times 10^{-4}, 2.29 \times 10^{-4}$, and $1.09 \times 10^{-3}$, respectively. On the other hand, the averages of the measurement errors in the $x$ direction using the original method are $2.05 \times 10^{-4}, 1.63 \times 10^{-3}$, $5.21 \times 10^{-3}$, and $1.64 \times 10^{-3}$, respectively. The averages in the $y$ direction are $1.10 \times 10^{-3}, 1.81 \times 10^{-2}, 3.60 \times 10^{-2}$, and $1.13 \times 10^{-2}$, respectively. The averages in the direction $x$ using the proposed method are $1.86 \times 10^{-4}, 2.04 \times 10^{-4}, 8.75 \times 10^{-5}$, and $2.54 \times 10^{-4}$, respectively. The averages in the direction $y$ are $1.05 \times 10^{-3}, 9.48 \times 10^{-4}, 2.99 \times 10^{-4}$, and $1.49 \times 10^{-3}$, respectively. It indicates that the measurement errors in two directions using the method with the constraint of 2D projective lines and $3 \mathrm{D}$ world points have higher accuracy than the original method.
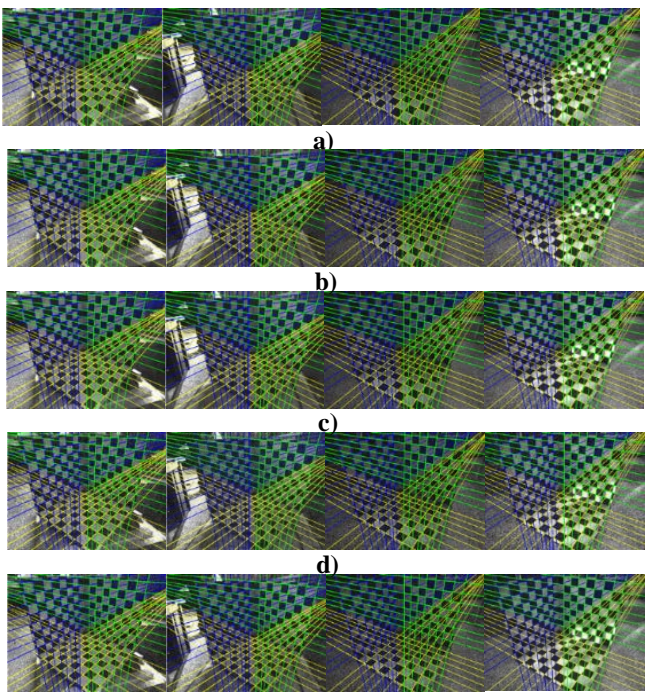

e)

Fig.6. Line recognition results of Fig.3.a) - d) with noises.

a) the noise is $0.0005, b)$ the noise is 0.005 , c) the noise is 0.001 , d) the noise is 0.02 , e) the noise is 0.01 .

Five levels of Gaussian noises are added to the original images to analyze the influence of the noise on the experiment results of the point-based calibration and the line-based calibration. Based on two calibration methods, we reproject 48 lines that are derived from three planes of the $3 \mathrm{D}$ calibration board. The number of $2 \mathrm{D}$ projective lines varies from 8 to 48 with an interval of 8 in the experiments. The line extraction results are shown in Fig.6., in which the variances of the noises are $0.0005,0.005,0.001,0.02$, and 0.01 , respectively. The relationship between the noise level, the number of lines and the average errors using the point-based calibration and the line-based calibration are 
illustrated in Fig.7. The average errors are identified by the root-mean-square errors of the adopted lines. In order to observe the noise data more conveniently, Gaussian noise and average errors are shown by the denary logarithm. In Fig.7.a)-Fig.7.d), average logarithmic errors of both calibration methods decrease when Gaussian noise is on the decline. In the direction of the lines' number, the average logarithmic errors of these two calibration methods gradually diminish when the number of adopted lines in the experiment increases from 8 to 48. It indicates that the calibration is more accurate with the increasing lines' number and decreasing noises. Moreover, it is obvious that the average errors generated from the proposed method are smaller than the point-based calibration method.

The results are compared in Table 3 . and Table 4. The variances of average errors using the proposed method in four images are $1.70 \times 10^{-5}, 1.39 \times 10^{-4}, 1.13 \times 10^{-4}$, and $4.06 \times 10^{-4}$, respectively. The variances of the original method are $9.07 \times 10^{-4}, 1.41 \times 10^{-3}, 5.90 \times 10^{-4}$, and $5.10 \times 10^{-3}$, respectively. As for the entire data using the proposed method, the smallest average errors in the images are $8.10 \times 10^{-4}, 1.70 \times 10^{-3}, 6.93 \times 10^{-4}$, and $2.60 \times 10^{-3}$, respectively. The biggest average errors are $2.14 \times 10^{-2}, 6.07 \times 10^{-2}$, $5.29 \times 10^{-2}$, and $8.27 \times 10^{-2}$ under the noise level of -3.301 and 48 projective lines. For the results using the original method, the smallest average errors are $1.90 \times 10^{-3}, 2.09 \times 10^{-2}$, $1.42 \times 10^{-2}$, and $2.10 \times 10^{-2}$, respectively. The biggest average errors are $1.34 \times 10^{-1}, 1.42 \times 10^{-1}, 9.88 \times 10^{-2}$, and $3.17 \times 10^{-1}$, respectively. The distributions of the errors show that the line-based calibration method is more stable for the noises than the original method.

Table 3. Results of variances of average errors.

\begin{tabular}{|c|c|c|}
\hline \multirow{2}{*}{ Image } & \multicolumn{2}{|c|}{ Variances of average errors } \\
\cline { 2 - 3 } & Point-based method & Line-based method \\
\hline $\mathrm{a}$ & $9.07 \times 10^{-4}$ & $1.70 \times 10^{-5}$ \\
\hline $\mathrm{b}$ & $1.41 \times 10^{-3}$ & $1.39 \times 10^{-4}$ \\
\hline $\mathrm{c}$ & $5.90 \times 10^{-4}$ & $1.13 \times 10^{-4}$ \\
\hline $\mathrm{d}$ & $5.10 \times 10^{-3}$ & $4.06 \times 10^{-4}$ \\
\hline
\end{tabular}

Table 4. Results of average errors under the noise of -3.301 .

\begin{tabular}{|c|c|c|c|}
\hline \multirow{2}{*}{\multicolumn{2}{|c|}{ Image }} & \multicolumn{2}{c|}{ Average errors } \\
\cline { 3 - 4 } \multicolumn{2}{|c|}{} & $\begin{array}{c}\text { Point-based } \\
\text { method }\end{array}$ & $\begin{array}{c}\text { Line-based } \\
\text { method }\end{array}$ \\
\hline \multirow{2}{*}{ a } & Smallest errors & $1.90 \times 10^{-3}$ & $8.10 \times 10^{-4}$ \\
\cline { 2 - 4 } & Biggest errors & $1.34 \times 10^{-1}$ & $2.14 \times 10^{-2}$ \\
\hline \multirow{2}{*}{ b } & Smallest errors & $2.09 \times 10^{-2}$ & $1.70 \times 10^{-3}$ \\
\cline { 2 - 4 } & Biggest errors & $1.42 \times 10^{-1}$ & $6.07 \times 10^{-2}$ \\
\hline \multirow{2}{*}{ c } & Smallest errors & $1.42 \times 10^{-2}$ & $6.93 \times 10^{-4}$ \\
\cline { 2 - 4 } & Biggest errors & $9.88 \times 10^{-2}$ & $5.29 \times 10^{-2}$ \\
\hline \multirow{2}{*}{ d } & Smallest errors & $2.10 \times 10^{-2}$ & $2.60 \times 10^{-3}$ \\
\cline { 2 - 4 } & Biggest errors & $3.17 \times 10^{-1}$ & $8.27 \times 10^{-2}$ \\
\hline
\end{tabular}

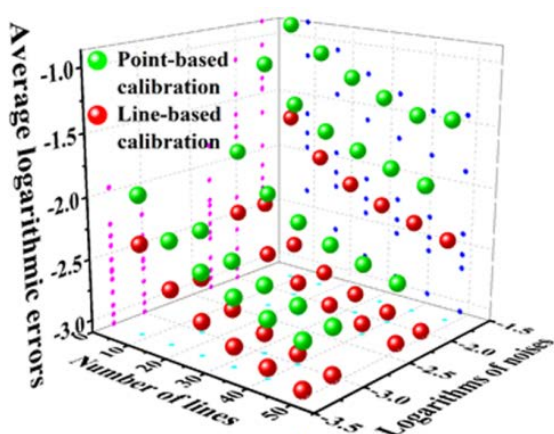

a)

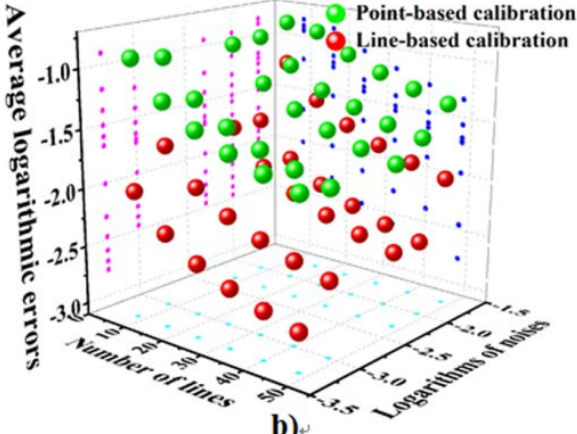

b)
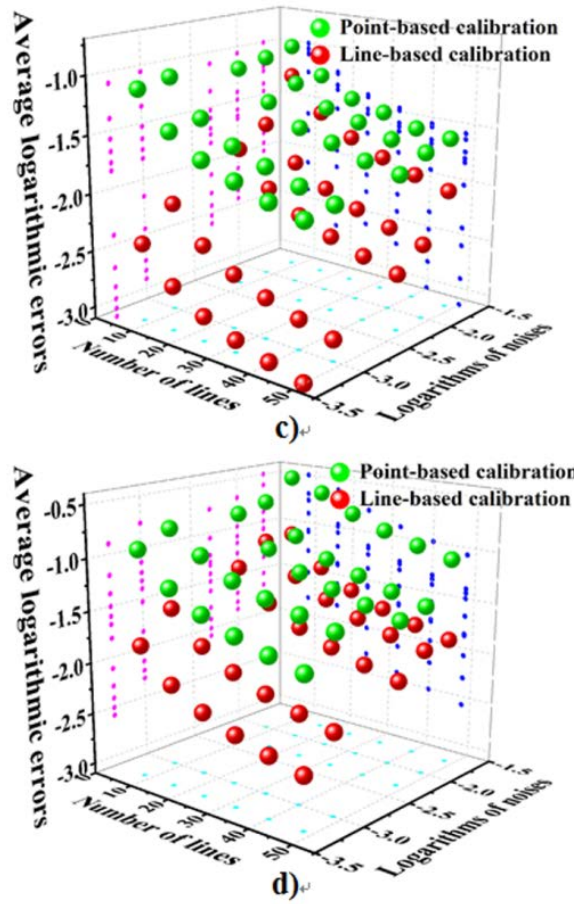

Fig.7. Average logarithmic errors related to the logarithms of noises and the number of lines in the point-based and line-based calibrations. a) - d) correspond to the results of Fig.3. and Fig.6.

\section{CONCLUSIONS}

In this work, we proposed an accurate method using the constraint of 2D line features and 3D world points for the camera calibration. We illustrate a calibration model considering the geometrical relationship among 3D points, 2D image plane and 2D projective lines. The transformation matrix of the camera can be solved by the singular value decomposition through the known coordinates of the $3 \mathrm{D}$ 
points and the coordinates of the projective lines extracted by the Hough transform. Furthermore, the experiments are conducted to compare the calibration precision and the noise immunity between the line-based calibration and the point-based calibration. We analyze the logarithms of the root-mean-square errors in the $x, y$ directions for different projective lines. The mean values of the root-mean-square errors using the proposed method are $7.69 \times 10^{-4}, 6.98 \times 10^{-4}$, $2.29 \times 10^{-4}$, and $1.09 \times 10^{-3}$. We also observe that the average logarithmic errors vary with the group number of the projective lines and different Gaussian noises. The variances of average errors using the proposed method are $1.70 \times 10^{-5}$, $1.39 \times 10^{-4}, 1.13 \times 10^{-4}$, and $4.06 \times 10^{-4}$, which are smaller than the original method. The smallest average errors using the proposed method are $8.10 \times 10^{-4}, 1.70 \times 10^{-3}, 6.93 \times 10^{-4}$, and $2.60 \times 10^{-3}$ in the images, respectively. The experiments demonstrate that the line-based calibration is able to improve the accuracy and robustness of the camera calibration in vision measurement. The 3D calibration board has the advantages of high precision and simple relationship between 3D points and 2D points. Therefore, we choose 3D calibration board and model the calibration process. However, the 3D calibration board is difficult to be made and preserved. In future work, 2D or 1D based methods considering line features are the directions for further development.

\section{ACKNOWLEDGMENT}

We gratefully acknowledge the supports of National Natural Science Foundation of China under Grant No. 51205164, Grant No. 51478204, Natural Science Foundation of Jilin Province under Grant No. 20150101027JC, and Jilin Province Science Foundation for Youths under Grant No. 20130522154JH.

\section{REFERENCES}

[1] Frollo, I., Krafčík, A., Andris, P., Přibil, J., Dermek, T. (2015). Circular samples as objects for magnetic resonance imaging-mathematical simulation, experimental results. Measurement Science Review, 15 (6), 313-318.

[2] Zhang, Y.P., Zimin, L.G., Ji, J., Ikezawa, S., Ueda, T. (2012). Real scene capturing using spherical single-element lens camera and improved restoration algorithm for radially variant blur. Optics Express, 20 (25), 27569-27588.

[3] Zhang, Q.S., Kamata, S. (2013). Improved color barycenter model and its separation for road sign detection. IEICE Transactions on Information and Systems, 96 (12), 2839-2849.

[4] Glowacz, A., Glowacz, A., Glowacz, Z. (2015). Recognition of monochrome thermal images of synchronous motor with the application of skeletonization and glassifier based on words. Archives of Metallurgy and Materials, 60 (1), 27-32.
[5] Huang, L., Zhang, Q.C., Asundi, A. (2013). Camera calibration with active phase target: Improvement on feature detection and optimization. Optics Letters, 38 (9), 1446-1448.

[6] Glowacz, A., Glowacz, A., Glowacz, Z. (2015). Recognition of thermal images of direct current motor with application of area perimeter vector and Bayes classifier. Measurement Science Review, 15 (3), 119-126.

[7] Cui, J.S., Huo, J., Yang, M. (2015). The circular mark projection error compensation in camera calibration. Optik, 126 (20), 2458-2463.

[8] Hong, Y.Z., Ren, G.Q., Liu, E.H. (2015). Non-iterative method for camera calibration. Optics Express, 23 (18), 23992-24003.

[9] Harding, K. (2008). Industrial metrology: Engineering precision. Nature Photonics, 2 (11), 667-669.

[10] Murawski, K. (2015). New vision sensor to measure and monitor gas pressure. Acta Physica Polonica A, 128 (1), 6-9.

[11] Kim, J.H., Koo, B.K. (2013). Linear stratified approach using full geometric constraints for 3D scene reconstruction and camera calibration. Optics Express, 21 (4), 4456-4474.

[12] Huang, L., Kemao, Q., Pan, B., Asundi, A.K. (2010). Comparison of Fourier transform, windowed Fourier transform, and wavelet transform methods for phase extraction from a single fringe pattern in fringe projection profilometry. Optics and Lasers in Engineering, 48 (2), 141-148.

[13] Zhang, Z.Y. (2000). A flexible new technique for camera calibration. IEEE Transactions on Pattern Analysis and Machine Intelligence, 22 (11), 1330-1334.

[14] Zhang, Z.Y. (2004). Camera calibration with one-dimensional objects. IEEE Transactions on Pattern Analysis and Machine Intelligence, 26 (7), 892-899.

[15] Ma, W.H., Dong, T., Tian, H., Ni, J.P. (2014). Line-scan CCD camera calibration in 2D coordinate measurement. Optik, 125 (17), 4795-4798.

[16] Vo, M., Wang, Z.Y., Hoang, T., Nguyen, D. (2010). Flexible calibration technique for fringe-projection-based three-dimensional imaging. Optics Letters, 35 (19), 3192-3194.

[17] Duan, H.X., Wu, Y.H. (2012). A calibration method for paracatadioptric camera from sphere images. Pattern Recognition Letters, 33 (6), 677-684.

[18] Ying, X.H., Zha, H.B. (2008). Identical projective geometric properties of central catadioptric line images and sphere images with applications to calibration. International Journal of Computer Vision, 78 (1), 89-105. 
[19] Cui, Y., Zhou, F.Q., Wang, Y.X., Liu, L., Gao, H. (2014). Precise calibration of binocular vision system used for vision measurement. Optics Express, 22 (8), 9134-9149.

[20] Li, K., Wang, Q., Wu, J., Yu, H.Y., Zhang, D.S. (2012). Calibration error for dual-camera digital image correlation at microscale. Optics and Lasers in Engineering, 50 (7), 971-975.

[21] Li, L.L., Zhao, W.C., Wu, F., Liu, Y., Gu, W. (2014). Experimental analysis and improvement on camera calibration pattern. Optical Engineering, 53 (1), 013104.

[22] Zhang, H.Y., Yang, F., Wu, Y.D., Paindavoine, M. (2009). Robust color circle-marker detection algorithm based on color information and Hough transformation. Optical Engineering, 48 (10), 107202.

[23] Xu, G., Sun, L.N., Li, X.T., Su, J., Hao, Z.B., Lu, X. (2014). Global calibration and equation reconstruction methods of a three dimensional curve generated from a laser plane in vision measurement. Optics Express, 22 (18), 22043-22055.
[24] Xu, G., Li, X.T., Su, J., Pan, H.D., Tian, G.D. (2011). Precision evaluation of three-dimensional feature points measurement by binocular vision. Journal of the Optical Society of Korea, 15 (1), 30-37.

[25] Leon, S.J. (2006). Linear Algebra with Applications (7th ed.). Prentice Hall.

[26] Walton, S., Hassan, O., Morgan, K. (2013). Reduced order modelling for unsteady fluid flow using proper orthogonal decomposition and radial basis functions. Applied Mathematical Modelling, 37 (20-21), 8930-8945.

[27] Chakraborty, B., Gonzalez, J., Roca, F.X. (2013). Large scale continuous visual event recognition using max-margin Hough transformation framework. Computer Vision and Image Understanding, 117 (10), 1356-1368.

[28] Dodge, Y. (2003). The Oxford Dictionary of Statistical Terms. Oxford University Press.

Received February 28, 2016. Accepted July 20, 2016. 